\title{
Efecto de polímeros sobre los niveles de IgAs anti Streptococcus mutans y la producción de dextranos de Streptococcus mutans autóctonos (estudio in vitro e in vivo)
}

\author{
Bojanich A*, Calamari SE**, Cornejo LS***, Barembaum S*, Virga C****, \\ Dorronsoro $\mathrm{S} * * *$
}

\section{RESUMEN}

Se evaluó el efecto de Quitosan de Alto Peso Molecular (QAPM) (0.25-0.50 g\% P/N; NaOH 1M) y Alginato de Sodio (Al-Na) 0.10-0.50 g\% P/ ; $\mathrm{CaCl}_{2} 1 \mathrm{M}$, sobre los niveles de IgAs anti Streptococcus mutans y la producción de dextranos.

Se estudió en saliva (in vitro) el efecto de los polímeros a 5', 8h y 24 h y en saliva extraída de diez sujetos sanos, (in vivo) a 0,1 y $3 \mathrm{~h}$ posteriores a la realización de un buche con polímero.

Los niveles de IgAs anti St mutans determinados por ELISA, no revelaron diferencias estadísticamente significativas con respecto al control $(p=0.05)$.

Se determinó la producción de dextranos en presencia de diferentes concentraciones de polímeros mediante espectrofotometría $(\lambda=520)$, utilizando de 1 a $5.10^{8} \mathrm{UFC} / \mathrm{ml}$ de St. mutans en caldo sacarosa $8,5 \%$. Los resultados mostraron reducción significativa, en la formación de dextranos respecto al control $(p<0.05)$, siendo de $32 \%$ para QAPM $0.25 \mathrm{~g} \%$ P/V y 66,8\% para QAPM $0.50 \mathrm{~g} \%$ P/N, en tanto que para Al-Na $0.10 \mathrm{~g} \%$ P/V fue del $30 \%$ y Al-Na $0.50 \mathrm{~g} \%$ P/N fue del $32 \%$.

Se concluye que QAPM y Al-Na , en razón de sus características físico-químicas que les permiten formar uniones tipo puente hidrógeno, y cadenas poliméricas que les confieren elasticidad suficiente para acceder a las distintas estructuras de la cavidad oral brindan expectativas para la investigación de nuevas formulaciones terapéuticas de aplicación odontológica.

Palabras claves: Quitosan de Alto Peso Molecular, Alginato de Sodio, IgAs anti-Streptococcus mutans, Dextrano, Streptococcus mutans.

\section{SUMMARY}

Effect of High Molecular Weight Quitosan (HMWQ) (0.25-0.50 g\% P/N, NaOH 1M) and Sodium Alginate (Na-Al) $\left(0.10-0.50 \mathrm{~g} \% \mathrm{P} / \mathrm{V}, \mathrm{CaCl}_{2} 1 \mathrm{M}\right.$, was evaluated on the levels of IgAs anti Streptococcus mutans and the production of dextran

It was studied in saliva (in vitro) the effect of the polymers to 5', $8 \mathrm{~h}$ and $24 \mathrm{~h}$ and in saliva from ten healthy subjects (study in vivo,) to 0,1 and $3 \mathrm{~h}$ after the execution of a mouth rinse with polymers. The levels of IgAs anti St mutans 
were determined by ELISA, they did not reveal statistically significant differences with regard to the control $(p=0.05)$. The dextran production was determined at different concentrations of polymers by means of espectrofotometria $(\lambda=520)$, using a suspension from 1 to 5.108 UFC $\mathrm{ml}$ of St. mutans in sucrose broth $8,5 \%$. The results showed significant reduction, to the formation of dextran with respect to the control $(\mathrm{p}<0.05)$, being for $32 \%$ to HMWQ $0.25 \mathrm{~g} \%$ $\mathrm{P} / \mathrm{V}$ and $66,8 \%$ for HMWQ $0.50 \mathrm{~g} \% \mathrm{P} / \mathrm{V}$, while for Na-Al $0.10 \mathrm{~g} \% \mathrm{P} / \mathrm{V}$ was $30 \%$ and Na-Al $0.50 \mathrm{~g} \% \mathrm{P}$ V was the $32 \%$.

It is concluded that QAPM and Al- Na, with regard to their characteristic physical and chemistry that permit to form hydrogen bond like, and polymeric chains that confer sufficient elasticity to agree to the different structures of the oral cavity, offer expectations for the therapeutic investigation of new formulations of deontological application.

Key words: Hight molecular chitosan, Sodium Alginate, IgAs anti-Streptococcus mutans, Dextran, Streptococcus mutans

Aceptado para publicación: Mayo 2003.

* Jefe de Trabajos Prácticos, Cátedra de Introducción a la Química, Facultad de Odontología, Universidad Nacional de Córdoba.

** Profesor Adjunto, Cátedra de Introducción a la Química, Facultad de Odontología, Universidad Nacional de Córdoba.

*** Profesor Titular, Cátedra de Biología Celular, Facultad de Odontología, Universidad Nacional de Córdoba.

*** Profesor Titular, Cátedra de Introducción a la Química, Facultad de Odontología, Universidad Nacional de Córdoba.

**** Profesor Adjunto, Departmento de Farmacología y Terapeútica,Facultad de Odontología, Universidad Nacional de Córdoba.

Bojanich A, Calamari SE, Cornejo LS, Barembaum S, Virga C, Dorronsoro S. Efecto de polímeros sobre los niveles de IgAs anti Streptococcus mutans y la producción de dextranos de Streptococcus mutans autóctonos (estudio in vitro e in vivo). Av. Odontoestomatol 2003; 19-5: 225-232.

\section{INTRODUCCIÓN}

La caries dental constituye una de las enfermedades de mayor prevalencia en la cavidad bucal. Se trata de una patología de etiología multicausal en la que diversos factores participan directa o indirectamente produciendo condiciones favorables para el comienzo y desarrollo del proceso patológico. Se reconocen factores tanto intrínsecos como extrínsecos, entre los primeros se menciona el estado anatómico y funcional de los tejidos de la cavidad bucal, calidad y cantidad de saliva, y el sistema inmunológico; entre los segundos la condición socioeconómica y cultural, hábitos y conductas de higiene bucal, elevado consumo de azúcares fermentables en alimentos y bebidas, disponibilidad de sustratos requeridos por microorganismos específicos, etc. La enfermedad se caracteriza por la desmineralización de los tejidos dentales, provocado por la acción de los ácidos orgánicos resultantes de la actividad metabólica de determinados microorganismos orales, siendo Streptococcus mutans la especie microbiana más fuertemente asociada con la caries dental (1-4).

Diversos estudios sobre las propiedades físicas y la actividad biológica de biopolímeros como Quitosan de alto y bajo peso molecular (QAPM y QBPM) y Alginato de sodio (Al-Na), evidencian que forman estructuras con capacidad de aglutinación, agregación y atrapamiento de microorganismos. QAPM con características policatiónicas, en pH ácidos ( $\mathrm{pH}: 5,5)$, se presenta soluble, cuando se eleva el $\mathrm{pH}$ a valores de alrededor de 7 adquiere estructuras más rígidas, en forma de red o malla, debido a que la fuerza iónica y las modificaciones del $\mathrm{pH}$ del medio son capaces de producir un cambio conformacional expresado mediante la formación de coloides estables (5-7). Mientras que $\mathrm{Al}-\mathrm{Na}$, carbohidrato hidrofilico, se comporta como un biomaterial aniónico, soluble en agua con capacidad de interactuar con cationes polivalen- 
tes como $\mathrm{Ca}^{++}$formando hidrogeles. Las formas insolubles resultantes del proceso físico-químico son capaces de inmovilizar especies microbianas como eubacteria, cianobacteria y hongos (8-10).

Estudios previos realizados por este equipo de investigación demostraron la efectividad de QAPM y Al-Na para agregar, atrapar e impedir la adherencia de Streptococcus mutans a perlas de hidroxiapatita tratadas con saliva y a células epiteliales bucales $(11,12)$.

La adherencia de los microorganismos a las superficies de la cavidad oral constituye la etapa inicial para la colonización y posterior desarrollo de infecciones. La misma depende de la características estructurales del microorganismo (adhesinas) y del polimorfismo de los receptores del huésped (glicoproteínas y proteínas salivales) que asientan en las diferentes localizaciones de la cavidad bucal (13-15).

Entre las glicoproteínas salivales que participan en la adherencia se reconocen la inmunoglobulina A secretora (lgAs), mucinas (MG1, MG2), proteínas ricas en prolinas (PRPA), fibronectina, amilasa, estaterina, cistatina y lactoferrina. Dichas moléculas sufren una modificación conformacional cuando son adsorbidas y exponen sitios específicos para interactuar con la proteínas bacterianas (16). Por su parte, las IgAs actúan como primer agente en la defensa inmunológica de la superficie de la mucosa oral interfiriendo la colonización de dichas superficies por parte de las células microbianas a través de distintos mecanismos: en forma conjunta favorecen la aglutinación bacteriana y se unen al antígeno soluble antes que el mismo pueda adsorberse a la mucosa y poseen actividad frente a componentes bacterianos específicos tales como toxinas, adhesinas y enzimas de superficie. En razón a ello, tanto la cuantificación como la valoración de la integridad de las estructuras de dicho anticuerpo constituye un valioso indicador del grado de susceptibilidad que presenta el huésped $(17,18)$.

Por otra parte la capacidad de adherencia de un microorganismo constituye en muchos casos un importante factor de virulencia. En el caso de Streptococcus mutans el proceso de adhesión se encuentra estrechamente relacionado con el metabolismo de la sacarosa, el cual es mediado por la acción del sistema enzimático: Glucosiltransferasas (GTFs), responsable clave en la síntesis extracelular de dextranos (polisacárido constituyente de la matriz de la placa dental). La sacarosa es la fuente natural de energía para el sistema GTFs, el cual es clave para catalizar la conversión de sacarosa a largas cadenas de polisacáridos $(19,20)$.

Toda interferencia en la actividad del sistema GTFS incidiría en la adherencia microbiana, afectando el avance del proceso patológico.

El objetivo de esta investigación es evaluar el efecto del QAPM y del Al-Na sobre los niveles de IgAs antiSt mutans presente en saliva in vivo e in vitro y el sistema GTFS a través de la producción de dextranos.

\section{MATERIALES Y MÉTODOS}

Saliva: se recogió saliva no estimulada proveniente de diez adultos sin caries activas, aproximadamente dos horas después de la última ingesta, mediante salivación directa en dos tubos de ensayo estériles, uno para el aislamiento de cepas autóctonas de Streptococcus mutans (St mutans) y el otro para la determinación de los niveles de IgAs anti-St mutans.

Microorganismos: a partir de las muestras de saliva se aislaron cepas autóctonas de St mutans utilizando un medio selectivo: Agar Mitis Salivarius-Bacitracina (DIFCO), incubándose a $37^{\circ} \mathrm{C}$ por $48 \mathrm{Hs}$ con $5 \% \mathrm{CO}_{2}$. Las cepas se identificaron por pruebas bioquímicas convencionales y por un kit comercial: API 20 Strep (BioMérieux, Francia) (21).

Polimeros: Quitosan de Alto Peso Molecular: (QAPM) (Sigma), purificado al $1 \mathrm{~g} \%$ P/N, deacetilado al $85 \%$, de peso molecular aproximado de $300 \mathrm{kD} \mathrm{y}$ una viscosidad de $280 \mathrm{cps}$, fue disuelto en ácido acético $0,1 \mathrm{~N}(\mathrm{pH}: 5,9)$.

Alginato de sodio ( Al-Na ) (Sigma), viscosidad del $2 \%$, a una concentración del $1 \mathrm{~g} \% \mathrm{P} / \mathrm{V}$.

Preparación del antígeno: las cepas de St mutans aisladas e identificadas, se suspendieron en caldo cerebro corazón (Biokar Diagnostics, Francia) y se 
concentraron mediante centrifugación a $3500 \mathrm{xg}$ durante 5'. Luego fueron lavadas dos veces con PBS $50 \mathrm{mM}$. El sedimento fue resuspendido en $1 \mathrm{~mL}$ de dicho buffer y sonicado 30s (Sonicar: amplitud 39\% Higth Intensity Ultrasonic Processor controlled 400 watt), posteriormente se centrifugó a 5000 xg y nuevamente se resuspendió en $1 \mathrm{~mL}$ de PBS y se determinó la concentración de proteínas totales según el método de Lowry et al (22). Se utilizó una alícuota con una concentración de proteínas de $15 \mu \mathrm{g} / \mathrm{mL}$.

Determinación de IgAs anti - St mutans de cepas autóctonas: las muestras de saliva se inactivaron por calentamiento a $56^{\circ} \mathrm{C}$ durante 30 s y se centrifugaron a $1000 \mathrm{xg}$, y los sobrenadantes se mantuvieron a $-20^{\circ} \mathrm{C}$ por un tiempo no mayor de una semana. Posteriormente en dichas muestras de ensayo se determinaron los niveles de IgAs anti St mutans por la técnica de ELISA, según descripción de Lehtonen (23).

Interacción de QAPM y Al-Na sobre los niveles de IgAs in vitro: se realizó de acuerdo a los criterios de procedimientos descriptos ( $\mathrm{a}$ y b) en trabajos previos donde se evaluó el fenómeno de atrapamiento, agregación y/o adhesión de los polímeros de estudio $(11,12)$.

En el procedimiento a), dos alícuotas de $2,5 \mathrm{ml}$ de saliva se le adicionaron los polímeros por separado, en ambos casos se utilizó la concentración inhibitoria mínima (CIM) $0.25 \mathrm{~g} \% \mathrm{P} / \mathrm{V}$ para QAPM y $0,10 \mathrm{~g}$ \% P/V para Al-Na ,según los criterios descriptos en trabajos previos $(11,12)$, y para producir el cambio conformacional, se agregó a las muestras con QAPM y Al-Na buffer fosfato $0,4 \mathrm{M} \mathrm{pH}: 7 \mathrm{y} \mathrm{CaCl}_{2} 1 \mathrm{M}$ respectivamente.

Se realizó una segunda experiencia, procedimiento b), en la que se provocó el cambio conformacional en primer lugar y posteriormente se puso en contacto con las muestras de saliva.

En ambas experiencias, después de realizar los procedimientos a) y b), se recogieron muestras a los 5 minutos, 8 hs y 24 hs. Dichas muestras fueron diluídas con buffer PBS (dilución 1/20), en las que se procedió a realizar la técnica de ELISA para evaluar los niveles de IgA: se usaron proteínas antigénicas (15 $\mu \mathrm{g} / \mathrm{mL}$ ), Anti-IgA humana (cadena a específica, preparada en conejo) (Sigma), fosfatasa alcalina conjugada a una dilución 1/1000 en NSS-PBS (Sigma) y tabletas de fosfato de paranitrofenol (Sigma) (23).

La absorbancia (DO) de las muestras se leyeron espectrofotométricamente a $405 \mathrm{~nm}$. Se utilizó un control sin inclusión de los polímeros. Cada muestra fue procesada por cuadriplicado.

Interacción de QAPM y Al-Na sobre los niveles de IgAs. in vivo: la población de estudio consistió en dos grupos de diez individuos, ambos sexos, sin caries activas, quienes realizaron buches con QAPM $0.50 \mathrm{~g} \mathrm{\%} \mathrm{P/V} \mathrm{o} \mathrm{con} \mathrm{Al-Na} 0.50 \mathrm{~g} \mathrm{\%} \mathrm{P/V} \mathrm{durante} 1$ minuto. Posterior al enjuagatorio, se recogió saliva a tres tiempos diferentes: 0,1 y 3 horas. Las muestras obtenidas se inactivaron a $56^{\circ} \mathrm{C}$ durante 30 s, se centrifugaron a $1000 \mathrm{xg}$ y el sobrenadante se diluyó con buffer PBS (1/20) para la determinación de los niveles de IgAs anti - St mutans por ELISA (23). Se utilizó un control sin inclusión de los polímeros. Cada muestra fue procesada por cuadriplicado.

Determinación de dextranos: la producción de dextranos se determinó por espectrofotometría a 520 $\mathrm{nm}$ utilizando una suspensión de 1 a 5 × $10^{8} \mathrm{UFC} / \mathrm{ml}$ de St mutans en caldo sacarosa al $8,5 \%$ con el agregado de biopolímeros: QAPM al $0.25 \mathrm{~g} \%$ P/V y 0.50 $\mathrm{g} \% \mathrm{P} / \mathrm{V}$ y Al-Na al $0.10 \mathrm{~g} \%$ P/V y $0.50 \mathrm{~g} \%$ P/V. Dicha suspensión se incubó por 7 días a $37^{\circ} \mathrm{C}$ y con $5 \%$ de $\mathrm{CO}_{2}$. Se usó como control la misma suspensión en ausencia de los biopolímeros. Al cabo de los 7 días se provocó el cambio conformacional de los biopolímeros con el agregado de buffer fosfato $0,4 \mathrm{M}(\mathrm{pH}: 7)$ al QAPM y $\mathrm{CaCl}_{2} 1 \mathrm{M}$ al Al-Na. Para separar las células microbianas del producto enzimático, se centrifugó por 5 minutos a $4000 \mathrm{xg}$. Se tomó del sobrenadante $1 \mathrm{ml}$ y se dividió en partes iguales en dos tubos. Se agregó $4.5 \mathrm{ml}$ de acetato de sodio (10\%) a ambos tubos y luego $6 \mathrm{ml}$ y $12.5 \mathrm{ml}$ de etanol (95\%) a uno y otro tubo respectivamente. Se mezcló suavemente por inversión y se dejó reposar hasta las 3 horas. Se consideró la producción de dextranos cuando se observó turbidez u opalescencia en ambos tubos, la cual se midió por densidad óptica (DO). Los ensayos se repitieron en tres momentos diferentes y por triplicado (21).

Análisis estadístico: los resultados obtenidos fue- 
ron procesados estadísticamentre con el test $\mathrm{t}$ Student's para muestras apareadas y no apareadas, aplicando un nivel de significación de $\mathrm{p} \leq 0,05$.

\section{RESULTADOS}

Como se muestra en la Tabla I, tanto QAPM como Al$\mathrm{Na}$ no produjeron variaciones significativas $(p=0,05)$ en los niveles de IgA anti-St mutans según los procedimientos a) y b) en los que se consideraron diferentes tiempos y condiciones del estudio in vitro.

Asimismo al analizar la interacción entre los biopolímeros y los niveles de IgA anti-St mutans in vivo, no se observaron variaciones significativas para un $p=0,05$ en los diferentes tiempos del ensayo (Tabla II).

El efecto de los polímeros sobre la producción de dextranos, expresado en DO, se observa en el Gráfico 1. La incidencia de los compuestos ensayados a diferentes concentraciones sobre dicho polisacárido extracelular, reveló diferencias estadísticamente significativas con relación al control $(\mathrm{p}<0,05)$.

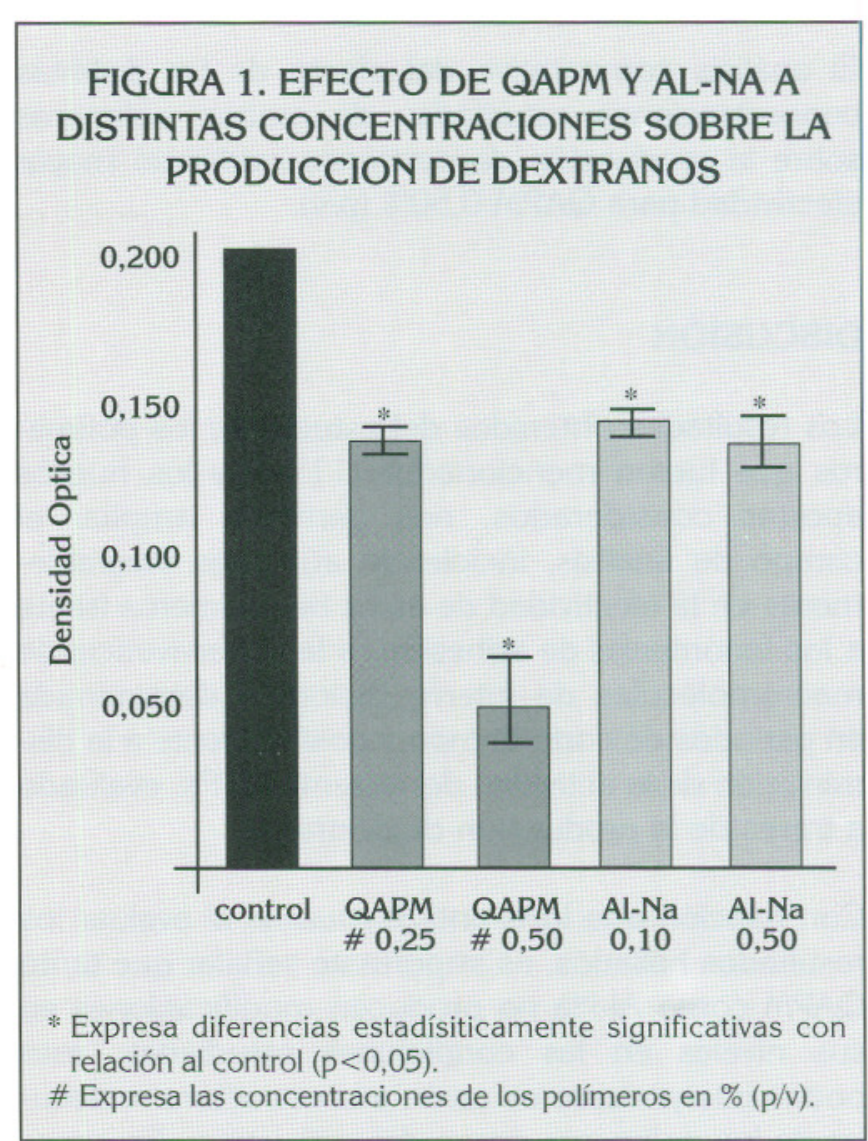

TABLA 1.- EFECTO DE QAPM Y AL-NA SOBRE NIVELES DE IgAs ANTI-ST MUTTANS. \# IN VITRO

\begin{tabular}{|c|c|c|c|}
\hline & 5 MINUTOS & $\begin{array}{c}\text { TIEMPO } \\
8 \text { HORAS }\end{array}$ & 24 HORAS \\
\hline a) Saliva + QAPM $0,25 \%+$ Buffer fosfato $0,4 M$ pH 7 & $984 \pm 350$ & $872 \pm 410$ & $824 \pm 280$ \\
\hline b) QAPM $0,25 \%$ + Buffer fosfato $0,4 M$ pH 7 + Saliva & $1110 \pm 435$ & $952 \pm 270$ & $932 \pm 450$ \\
\hline a) Saliva + Al-Na $0,10 \%+\mathrm{CaCl}_{2} 1 \mathrm{M}$ & $1022 \pm 341$ & $992 \pm 327$ & $810 \pm 370$ \\
\hline b) $\mathrm{Al}-\mathrm{Na} 0,10 \%+\mathrm{CaCl}_{2} 1 \mathrm{M}+$ Saliva & $980 \pm 326$ & $962 \pm 220$ & $940 \pm 290$ \\
\hline Control & $1270 \pm 280$ & $1238 \pm 231$ & $1198 \pm 122$ \\
\hline
\end{tabular}

TABLA 2.- EFECTO DE QAPM Y AL-NA SOBRE NIVELES DE IgAs ANTI-ST MUTANS. \# IN VIVO

\begin{tabular}{|lccc|}
\hline & & TIEMPO & 1 HORAS \\
\hline Saliva + QAPM 0,5\% & $1356 \pm 650$ & $1293 \pm 580$ & $1400 \pm 445$ \\
\hline Saliva + Al-Na 0,5\% & $1414 \pm 601$ & $1403 \pm 525$ & $1420 \pm 432$ \\
\hline Control & & $1560 \pm 497$ & \\
\hline $\begin{array}{l}\text { \# Los valores expresados en Unidades Arbitrarias representan la media aritmética. } \\
\pm \text { Desviación estándar. }\end{array}$ & & \\
\hline
\end{tabular}


análisis comparativo del efecto de las distintas concentraciones estudiadas de ambos polímeros sobre la producción de dextranos evidenció mayor efectividad para QAPM 0,50\% (p/v).

\section{DISCUSIÓN}

Los resultados obtenidos del estudio de los polímeros que fueran mencionados $(11,12)$ y los nuevos aportes considerados, nos permiten ampliar el campo de análisis, incidiendo aún más favorablemente en la efectividad de estos biopolímeros frente a los fenómenos de adhesión, a la supervivencia de macromoléculas de interés biológico determinada en períodos de corto tiempo y posiblemente a la disminución de la actividad del sistema GTFs, evaluado a través de la producción de dextranos.

Con relación a la IgAs anti-St mutans al evaluar los resultados hallados, es importante señalar que tanto QAPM como Al-Na no producen modificaciones en sus niveles en las condiciones estudiadas, esto podría interpretarse en razón a la estructura molecular de los polímeros como del anticuerpo. Es conocido que QAPM se comporta como un polímero policatiónico a $\mathrm{pH}$ próximos a 5, en tanto en un medio de $\mathrm{pH}$ cercano a 7, forma un coloide estable en el que predominan las interacciones hidrofóbicas, con actividad selectiva para diferentes compuestos y capacidad de atrapamiento y formación de complejos macromoluculares $(7,24,25)$.

Por su parte, Al-Na, polisacárido lineal, posee una estructura cargada negativamente capaz de interactuar con cationes polivalentes $\left(\mathrm{Ca}^{++}\right)$generando enlaces cruzados, lo que formaría un gel con alta capacidad de atrapar moléculas complejas $(9,10)$. A la vez resulta interesante remarcar que IgAs (PM $383 \mathrm{kD}$ ) posee un componente secretorio (PM 70 $\mathrm{kD}$ ) y un polipétido adicional (PM $15 \mathrm{kD}$ ), llamado cadena de unión $(\mathrm{J})$, señalada como responsable de la estabilidad molecular (26). Esta última característica sumada a las propiedades físico-químicas de los polímeros (carácter hidrofóbico, gel estable) podrían explicar la escasa interacción entre el polímero y el anticuerpo, manifestada por el comportamiento similar de la IgAs anti-St mutans en las diferentes condiones estudiadas.
Si bien la IgAs anti-St mutans juega un rol importante en el mantenimiento del equilibrio inmunológico del ambiente bucal, otros sistemas como factores de virulencia de los microorganismos operan antagónicamente y serían responsables de la ruptura de dicho equilibrio.

El sistema GTFs del St mutans juega un rol primordial y es considerado un factor de virulencia significativo de la bacteria, siendo la sacarosa la fuente natural de energía para este sistema enzimático el que cataliza la conversión de dicho disacárido en largas cadenas de polisacáridos (dextranos), constituyentes de la matriz de la placa dental $(1,20)$.

Los resultados del presente trabajo mostraron una diferencia estadísticamente significativa $(p<0,05)$ en la reducción de formación de dextranos con relación al control, siendo del 32\% para QAPM 0,25\% (p/v) y del $66,8 \%$ para QAPM 0,5\% (p/v), en tanto que del $30 \%$ para $\mathrm{Al}-\mathrm{Na} 0,10 \%(\mathrm{p} / \mathrm{v})$ y del $32 \%$ para $\mathrm{Al}-\mathrm{Na}$ $0,5 \%(\mathrm{p} / \mathrm{v})$. Las características físico-químicas de ambos compuestos y las del sistema GTFs modificarían el funcionamiento del mismo; puesto que el pKa de los compuestos bajo estudio es similar a los del bicarbonato salival y el $\mathrm{pH}$ en el cual gelifican es cercano a 7; mientras que el pH óptimo del sistema GTFs es próximo a 5, explicarían la disminución en la formación de dextranos.(9). El mayor efecto mostrado por QAPM al 0,5\% ( $\mathrm{p} / \mathrm{v})$, podría atribuirse a la presencia de un mayor número de cargas positivas que interactuarían con las cargas negativas de los glutamatos y aspartatos del sitio activo del sistema GTFs, no afectadas por el estado de gel del polímero, inactivando su actividad catalítica (27), argumento que fuera apoyado por otros autores al afirmar la capacidad de Quitosan de producir inactivación enzimática y quelación de iones metálicos del mismo modo que $\mathrm{Al}-\mathrm{Na}(7,24)$. Considerando que la síntesis de dextranos resulta de la actividad del sistema enzimático GTFs, la disminución del homopolisacárido extracelular de glucosa producido por las cepas autóctonas de Streptococcus mutans estudiadas en presencia de QAPM y de Al-Na sugeriría la incidencia de dichos polímeros en el sistema GTFs, considerado como un factor de virulencia de estos patógenos.

Además de ello, la capacidad de los polímeros para 
captar microorganismos, debido al proceso físicoquímico de gelificación que proporciona una gran superficie adhesiva apta para el atrapamiento de los mismos $(7,9,24)$, también afectaría la cantidad de dextranos formados.

Por lo anteriormente expuesto, se concluye que en relación al mantenimiento de los niveles de IgAs, a la disminución de la producción de dextranos y a las características físico-químicas de los biopolímeros (grupos funcionales capaces de formar uniones tipo puente hidrógeno, cargas aniónicas y catiónicas y cadenas poliméricas que le confieren elasticidad suficiente para acceder a las distintas estructuras de la cavidad oral) QAPM y Al-Na nos brindan expectativas para futuras investigaciones direccionadas a nuevas formulaciones aplicables en odontología.

\section{AGRADECIMIENTOS}

Esta investigación fue subsidiada por Agencia Córdoba Ciencia y Secretaría de Ciencia y Técnica de la Universidad Nacional de Córdoba.

\section{BIBLIOGRAFÍA}

1. Bowen, W HE Birkhed, D. Dental caries: dietary and microbiology factors. In Granat, LE Mc Hugh W D. ed. Systematized prevention of oral disease: theory and practice. Boca Raton, CRC, 1986, p 19-41.

2. Gibsona, S, Williamsb, S. Dental caries in Preschool children: Associations with Social Class, Toothbrushing Habit and Consumption of Sugars and sugar-containing Foods. Caries Res 1999; 33: 101-3.

3. Johansson Y, Ericson T,Steen L. Studies on the effect of diet on saliva composition and caries development. The effect of fasting on saliva composition. J Nutr 1984; 114: 2010-20.

4. van Houte J. Role of Micro-organisms in caries etiology. J Dent Res 1994; 73 (3): 672-81.

5. Muzzarelli RA., Biochemical significance of exo- genous chitins and chitosans in animal and patients. Carbohydr Polym 1993; 20:7-16.

6. Koide S.S. Chitin-Chitosan: properties, benefits and risks. Nutrition Res 1998; 18: 1091-101.

7. Muzzarelli RA., Ilari P., Petrarulo M. Solubility and structure of $\mathrm{N}$-carboxymethyl chitosan. Int J Biol Macromol 1994; 16: 177-80.

8. Barembaum S, Virga C, Bojanich A, Cornejo L, Calamari S, Ponton J, Dorronsoro S. Efecto de quitosan y alginato de sodio sobre la adherencia de $\mathrm{C}$ albicans autóctonas a células epiteliales bucales. In vitro. Medicina oral. (En prensa).

9. Linton JD, Ash-Huybretchts L. Biomaterials Novel materials from biological Sources: Algal Alginates 1987; p 309-31. Ed David Byrom, EEUU Stockton Press.

10. Klock G., Pfeffermann A. Biocompatibility of mannuronic acidic-rich alginates. Biomaterials 1997; 18 (10): 707-13.

11. Virga C, Cornejo LS, Calamari SE, Bojanich MA, Landa C, Beltramo D,Dorronsoro de Cattoni ST, Efecto de polímeros en la agregación y adherencia sobre hidroxiapatita de cepas autóctonas de St mutans y C albicans. Revista Vasca de Odontoestomatología 2001; 11 (2): 30-40.

12. Dorronsoro de Cattoni S T, Virga C, Calamari S, Bojanich A, Cornejo L, Barembaum S, Estudio comparativo del efecto de Quitosan y Alginato de sodio sobre la adherencia de St mutans autóctonos a células epiteliales y a perlas de Hidroxiapatita artificial. Estudio in-vitro. Revista Vasca de Odontoestomatología 2001;11 (4): 28-37.

13. Gibbons RJ. Bacterial adhesion to oral tissues. A model for infectious diseases. J Dent Res 1989; 68 (5): 750-60.

14. Gibbons RJ. Role of adhesion in microbial colonization of host tissues: a contribution of oral microbiology. J Dent Res 1996; 75 (3): 866-70.

15. Levine M J: Salivary macromolecules. A structu- 
re, function synopsis. Annals of the New York Academy of Sciences 1993; 694: 11-6.

16. Carlén A, Bratt P, Stenudd C, Olsson J, Stromberg N. Agglutinin and Acidic Proline-rich Protein Receptor Patterns May Modulate Bacterial Adherence and Colonization on Tooth Surfaces. J Dent Res 1998; 77 (1): 81- 90.

17. Williams RC, Gibbons RJ. Inhibition of bacterial adherence by secretory immunoglobulin: a mechanism of antigen disposal. Science 1972; 177: 697-99

18. Walker WA, Isselbacher KJ, Block Kj. Intestinal uptake of macromolecules. Science 1972; 177: 608-10.

19. Hamada S, Slade HD. Biology, immunology and cariogenicity of Streptococcus mutans. Microbiol Rev 1980; 44: 331-4.

20. Loesche WJ. Role of Streptococcus mutans in human dental decay. Microbiol Rev 1986; 50: 353-80.

21. Bantar C, Roncoroni AJ, Smayevsky J y Bianchini H. Evaluación del uso de un esquema de pruebas bioquímicas para tipificar estreptococos del grupo "viridans" y Streptococcus bovis. Infectología y Microbiología Clínica 1990; Vol2, 2: 48-54.

22. Lowry OH, Rosenbrough NJ, Farr AL, Randall RJ. Protein measurement with Folin phenol reagent. J Biol Chem 1951; 193: 265-75.
23. Lehtonen OP, Grahn EE, Sthalberg TH, Laitinen IA. Amount and avidity of salivary and serum antibodies against Streptococcus mutans in two groups of human subjects with different dental caries susceptibility. Infect Immun 1984; 43: 308-13.

24. Muzzarelli R., Tafani F., Emanuelli M. N(Carboxymethylidene) chitosans and $\mathrm{N}$ (Carboxymrthyl)-chitosans: novel chelating polyampholytes obtained from chitosan glyoxylate. Carbohydrate Res 1982; 107: 199-214.

25. Mckay G., Blair H.S., Findon A. Sorption of metal ions by chitosan, in: immobilization of ions by bio-sorption. Eng. Chem Res. 1998; 37: 145463.

26. Roitt I M. Essencial Immunology. In Structure of the Antibody Molecule and Immunoglobulin Genes. 9 ed; Oxford; Malden, MA: Blackwell Science 1997, p 3: 1-3:42.

27. Devulapalle K S and Mooser G. Glucosyltransferase Inactivation Reduces Dental Caries. J Dent Res 2001; 80 (2): 466-9.

\section{CORRESPONDENCIA}

Dra. Susana Dorronsoro

Haya de la Torre S/N .Agencia Postal 4. CP 5000

Facultad de Odontología- Ciudad Universitaria

Córdoba- ARGENTINA

e-mail:susu@ odo.unc.edu.ar 